\title{
Mechanism of Wire Explosion Plasma Acceleration in Strong Axial Magnetic Field
}

\author{
Yu.E. Adamian* and G.A. ShneERson \\ Saint-Petersburg State Polytechnical University \\ Politechnicheskaya st. 29, 195251 Saint-Petersburg, Russian Federation
}

\begin{abstract}
In previous wire explosion experiments significant rise of plasma expansion speed is registered when strong axial magnetic field $(50 \mathrm{~T})$ is applied. Sharp drop of media conductivity takes place in peripheral area because of density decrease. It is shown in this work that the conductivity drop is the main cause of plasma acceleration. During plasma expansion in axial field azimuthal current is induced. In plasma layer losing conductivity takes place azimuthal current energy release. As a result, pressure shock is formed and low-density external plasma layer is accelerated.
\end{abstract}

PACS numbers: 47.65.-d

\section{Introduction}

Experiments on wire explosion in vacuum in strong axial magnetic field were carried out in recent years. Vacuum chamber containing exploding wire was placed into solenoid, generating axial magnetic field. Wire explosion started at first maximum of axial field. It was observed that plasma boundary could move across the axial magnetic field with velocity up to $20 \mathrm{~km} / \mathrm{s}$ [1]. If the axial field is absent, the speed of expansion is substantially lower. It is known that axial field can decelerate the expansion of media with constant conductivity. Therefore, we suppose sharp change of media conductivity at wire periphery as main factor of peripheral plasma acceleration.

\section{Energy transition into media at sharp conductivity drop and acceleration of plasma external layer}

Geometry of experiment is schematically displayed in Fig. 1. Here $B_{z}, \delta_{z}$ - axial components, $B_{\varphi}, \delta_{\varphi}-$ azimuthal components of magnetic field and current density. Azimuthal current density $\delta_{\varphi}$ is induced at radial motion of media in axial field. Estimated mechanism of the axial field effect is the following. In expansion starting azimuthal eddy current is generated in external zone of wire. If speed of energy deposition to the wire is not high enough for media ionisation, sharp drop of the conductivity in external plasma layer [2] takes place. This layer is not affected by electromagnetic forces and media can expand free. Simultaneously certain part of induced

\footnotetext{
* corresponding author; e-mail: integr@delfa.net
}

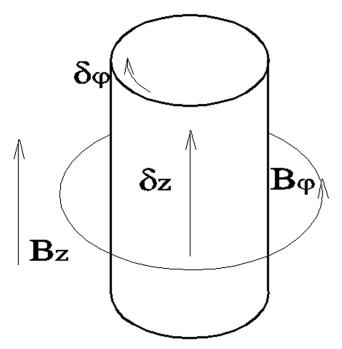

Fig. 1. Geometry of wire explosion in axial magnetic field.

azimuthal current energy is transforming into Joule heating in layer losing conductivity. It can lead to additional plasma heating and rise of plasma flow velocity. There are three characteristic points in flow area. The first of them - point 1 (its coordinate is $r_{1}$ in Fig. 2) where the density at some moment gets characteristic value $\rho^{\prime} \ll \rho_{0}$ at which sharp drop of the conductivity begins $\left(\rho_{0}-\right.$ initial media density). After beginning of this process point 2, where the density is equal to $\rho^{\prime}$ moves left from point 1 . To the right from point 1 density of the media is lower than characteristic value $\rho^{\prime}$ and conductivity is lost. For further estimations let us consider that to the left of point 2 the media is held by electromagnetic forces because of its high conductivity. Point 3 where the density reaches zero moves to the right with speed $u(3)$ corresponding to the media flow speed near this point. It is possible to use self-similar solution [3] for modelling of media expansion to vacuum.

According to this solution relative speed between points 1 and 2 is equal to adiabatic speed in point 2 


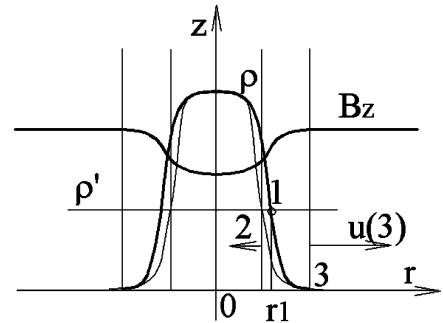

Fig. 2. Characteristic points in flow area.

$$
\frac{\mathrm{d} S}{\mathrm{~d} t}=c_{\mathrm{s}}=\sqrt{\frac{\gamma P(2)}{\rho(2)}},
$$

where $S$ is distance between points 1 and $2, P(2)$ is the pressure in point $2, \rho(2)=\rho^{\prime}$. Point 3 moves relatively to point 2 with the speed

$$
u(3,2)=[2 /(\gamma-1)] c_{\mathrm{s}} \text {. }
$$

According to self-similar solution increment of volumetric thermal energy can be estimated. Corresponding pressure increment in point 1 is

$$
\Delta P(1)=\theta S^{2} \delta(1)^{2} \mu_{0}(\gamma-1) .
$$

In this formula $\delta(1)$ is the azimuthal current density in point $1, \theta$ - non-dimensional coefficient characterizing sharpness of conductivity drop near threshold. In self-similar estimation $\theta=$ const. Speed of low-conductivity zone expansion can be determined as

$$
\frac{\mathrm{d} S}{\mathrm{~d} t}=\sqrt{\left[P_{0}+\theta S^{2} \mu_{0} \delta(1)^{2}(\gamma-1)\right] \frac{\gamma}{\rho^{\prime}}} .
$$

Here $P_{0}$ - initial pressure. Solution of this equation gives $S(t)$ and pressure in the point 1

$$
\begin{aligned}
& S(t)=\sqrt{\frac{P_{0}(\gamma-1)}{\mu_{0} \delta(1)^{2} \theta} \operatorname{sh}\left(t / t_{0}\right),} \\
& P(1)=P_{0}\left[1+\operatorname{sh}^{2}\left(t / t_{0}\right)\right] .
\end{aligned}
$$

In these formulae characteristic time is

$$
t_{0}=\sqrt{\frac{\rho^{\prime}}{\gamma(\gamma-1) \theta \mu_{0} \delta(1)^{2}}} .
$$

This parameter can be interpreted in simplest way in case of axial field displacement from plasma cylinder due to media expansion. In this case $\delta(1) \approx\left(B_{\mathrm{e}}-B_{\mathrm{i}}\right) / \mu_{0} r_{1}$, where $B_{\mathrm{e}}$ and $B_{\mathrm{i}}$ - field inductions outside and inside the cylinder of radius $r_{1}$. Then $t_{0}$ can be represented as

$$
t_{0}=r_{1} /\left\{[\gamma(\gamma-1)]^{0.5} \theta V_{\mathrm{A}}\right\},
$$

where $V_{\mathrm{A}}=\left(B_{\mathrm{e}}-B_{\mathrm{i}}\right) / \sqrt{\mu_{0} \rho^{\prime}}$ - Alfvén speed corresponding to inductance $B_{\mathrm{e}}-B_{\mathrm{i}}$.

In recent experiments on wire explosion in axial field with induction $50 \mathrm{~T}$ in accordance with diamagnetic signal measurements this value is $2-4 \mathrm{~T}$. According to used conductivity models $\theta \approx 10^{3}$ at radius $r_{1}=10^{-3} \mathrm{~m}$, correspondingly $t_{0} \approx 10^{-8}$. At free expansion the front (3) moves with the velocity $u(3)=2 c_{\mathrm{s}}(1) /(\gamma-1)$. This estimation following from self-similar solution shows that Joule heating of the media can lead to additional expan- sion velocity rise. Increase of thermal energy $\Delta q^{\prime}(1)$ can be estimated using formulae displayed above for initial stage of the process when $t<t_{0}$ and $\operatorname{sh}\left(t / t_{0}\right) \approx t / t_{0}$. This increase is proportional to $\left(t / t_{0}\right)^{2}$ :

$$
\Delta q^{\prime} \approx \frac{P_{0}}{\gamma-1}\left(\frac{t}{t_{0}}\right)^{2} .
$$

Along with rise of the axial field induction the characteristic time $t_{0}$ is going down and energy release rises. Increase of local sound speed, acceleration of the plasma boundary and partial transition of released thermal energy into kinetic are consequences of the process described. Described mechanism is similar to operation of inductive energy store, switched with conductor, losing its conductivity. During commutation process part of magnetic field energy is released in the switch. More detailed study of this phenomenon is out of self-similar solution limitations and can be conducted by computer simulations using artificial dependences of conductivity vs. density.

\section{Results of numeric modeling}

To illustrate this phenomenon we solved numerically simplified one-dimensional problem of plasma cylinder with given thermal energy expansion into vacuum in magnetic field $B_{z}=50 \mathrm{~T}$. Ideal gas approximation and artificial model of the conductivity sharply dropping with density decrease were used. Conductivity was approximated by the following formula:

$$
\sigma=\left(\sigma_{0} / \pi\right)\left[\arctan \left(\theta_{\mathrm{m}}\left(\rho / \rho_{0}-\xi\right)\right)+\pi / 2\right],
$$

where $\sigma_{0}, \rho_{0}$ - initial conductivity and density of media, $\theta_{\mathrm{m}}$ - defines sharpness of conductivity drop. Number $\xi<1$ characterizes relative density value corresponding to threshold of conductivity drop. For the comparison analogous calculation for model of constant conductivity $\sigma=\sigma_{0}$ was done. Calculations were conducted under following conditions: $\theta_{\mathrm{m}}=700, \sigma_{0}=10^{4} \Omega^{-1} \mathrm{~m}^{-1}, \xi=0.1$. Initial temperature $k T=5 \mathrm{eV}$, density $\rho_{0}=40 \mathrm{~kg} / \mathrm{m}^{3}$. Calculated profiles of azimuthal current density, media density and local plasma velocity for time moment $100 \mathrm{~ns}$ after expansion beginning are displayed in Fig. 3a. Here 1 is media density $\rho, 2$ - azimuthal current density $\delta, 3-$ local plasma velocity $u$.

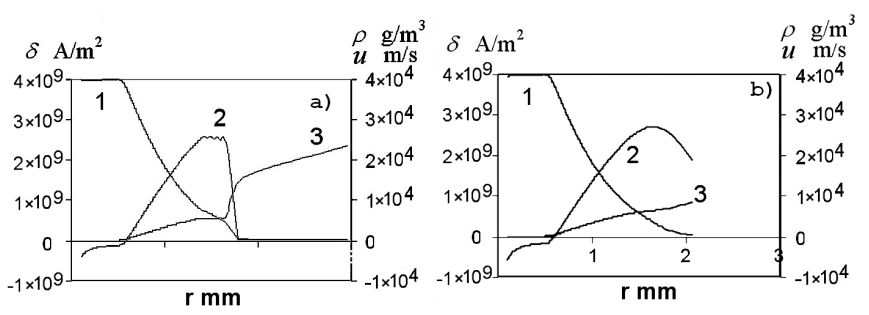

Fig. 3. Calculated profiles of media density (1), azimuthal current density (2) and local media velocity (3) in expansion process with variable (a) and constant (b) conductivity. 
In Fig. 3b results for constant conductivity model are shown. It can be seen that with model of conductivity drop sharp plasma acceleration takes place in zone of conductivity drop. Further additional velocity rise on plasma periphery is caused by adiabatic expansion into vacuum. To the contrary, with constant conductivity plasma motion is reduced by axial magnetic field because of the Lorentz force action.

\section{Conclusion}

Wire explosion in strong axial magnetic field can be accompanied by external plasma layer acceleration. Supposed mechanism of the effect is based on known dependences of plasma conductivity on density. Induced azimuthal current is generated during media expansion in axial field. It reduces axial field induction inside the exploding wire. Simultaneously, media density drops in the periphery due to expansion into vacuum. In accor- dance with known conductivity models, sharp drop of the conductivity takes place if density is reduced several times in comparison with solid state. In plasma layer losing conductivity takes place an azimuthal current energy release. It is analogous to operation of inductive energy store, switched with conductor breaking the circuit. Described mechanism can qualitatively explain the experimental data.

\section{References}

[1] Yu.E. Adamian, V.M. Vasilevskiy, S.N. Kolgatin, G.A. Shneerson, Zh. Tech. Fiz. 69, 121 (1999) (in Russian); Technical Phys. 44, 588 (1999).

[2] Yu.D. Bakulin, V.F. Kouropatenko, A.V. Louchinsky, Zh. Tech. Fiz. 46, 1963 (1976) (in Russian) (Sov. Phys.-Technical Phys. 20, 1963 (1976)).

[3] I.B. Chekmarev, Prikladnaya Matematika $i$ Mekhanika 2, 382 (1960) (in Russian). 